\title{
Parametric Amplification of Vacuum Fluctuations in a Spinor Condensate
}

\author{
C. Klempt, ${ }^{1}$ O. Topic, ${ }^{1}$ G. Gebreyesus,${ }^{2}$ M. Scherer, ${ }^{1}$ T. Henninger, ${ }^{1}$ P. Hyllus, ${ }^{3}$ W. Ertmer, ${ }^{1}$ L. Santos, ${ }^{2}$ and J. J. Arlt ${ }^{4}$ \\ ${ }^{1}$ Institut für Quantenoptik, Leibniz Universität Hannover, D-30167 Hannover, Germany \\ ${ }^{2}$ Institut für Theoretische Physik, Leibniz. Universität Hannover, D-30167 Hannover, Germany \\ ${ }^{3}$ BEC-INFM, Dipartimento di Fisica, Università di Trento, Via Sommarive 14, I-38050 Povo, Italy \\ ${ }^{4}$ QUANTOP, Department of Physics and Astronomy, University of Aarhus, $8000 \AA$ Arhus C, Denmark \\ (Received 8 October 2009; revised manuscript received 2 April 2010; published 12 May 2010)
}

\begin{abstract}
Parametric amplification of vacuum fluctuations is crucial in modern quantum optics, enabling the creation of squeezing and entanglement. We demonstrate the parametric amplification of vacuum fluctuations for matter waves using a spinor $F=2{ }^{87} \mathrm{Rb}$ condensate. Interatomic interactions lead to correlated pair creation in the $m_{F}= \pm 1$ states from an initial $m_{F}=0$ condensate, which acts as a vacuum for $m_{F} \neq 0$. Although this pair creation from a pure $m_{F}=0$ condensate is ideally triggered by vacuum fluctuations, unavoidable spurious initial $m_{F}= \pm 1$ atoms induce a classical seed which may become the dominant triggering mechanism. We show that pair creation is insensitive to a classical seed for sufficiently large magnetic fields, demonstrating the dominant role of vacuum fluctuations. The presented system thus provides a direct path towards the generation of nonclassical states of matter.
\end{abstract}

Parametric amplification of vacuum fluctuations in nonlinear media [1] plays a crucial role in the investigation and application of nonclassical states of light. These states have revolutionized the field of quantum optics in the past decades. Since the first observation of squeezed light [2], these nonclassical states of light have become a valuable tool in modern optics, e.g., for the enhancement of modern interferometers [3]. Similarly, the production of entangled photon pairs [4] has triggered a series of fundamental tests of quantum mechanics $[5,6]$ and has many possible applications for quantum computing [7]. The tools developed for the production and manipulation of ultracold neutral atoms now bring many of these seminal investigations within the scope of experiments with matter waves. In this sense, the production of number-squeezed BoseEinstein condensates (BECs) [8,9] and spin squeezed thermal clouds [10] has been demonstrated using the inherent Kerr nonlinearity in BECs.

Bose-Einstein condensates of atoms with nonzero spin provide a promising method to generate nonclassical matter waves. The atoms in this work are prepared with a spin projection $m_{F}=0$ (represented by $|0\rangle$ ) onto the magnetic field axis. A collision of two atoms in $|0\rangle$ can form a pair of correlated atoms in $|+1\rangle$ and $|-1\rangle$. Formally, this process is exactly equivalent to photon pair creation in a nondegenerate optical parametric amplifiers [11]. In this analogy, the large BEC in $|0\rangle$ resembles a coherent pump, the nonlinear interactions in the BEC serve as an equivalent of the nonlinear crystal, and the produced atoms in $| \pm 1\rangle$ play the role of signal and idler. Pair production leads to an exponential amplification of the population in $| \pm 1\rangle$, which resembles the gain of an optical parametric amplifier. The corresponding pair creation rate is governed by three competing energy scales: the quadratic Zeeman energy (QZE), the interatomic interactions, and the external trap. In particular, the external confinement can lead to strong, magnetic field dependent spin resonances [12].

Similar to its optical counterpart, the output of the matter-wave amplifier depends crucially on the input state. An ideal input would be provided by the vacuum state $|\mathrm{vac}\rangle$, which is characterized by the absence of atoms in $| \pm 1\rangle$, and may be visualized as an ideally fixed input spin orientation of the $|0\rangle$ BEC. In this sense, vacuum fluctuations then result from the quantum uncertainty of the spin orientation. However, it is experimentally unavoidable to spuriously produce few single atoms in $| \pm 1\rangle$ which correspond to a coherent classical input for the amplifier. These classical seed atoms arise from tiny tilts of the spin orientation of the $|0\rangle$ BEC due to radio frequency (rf) or magnetic field noise and thus share the spatial wave function of the original $|0\rangle \mathrm{BEC}$.

In this Letter, we demonstrate the parametric amplification of vacuum fluctuations for matter waves using a spinor $F=2{ }^{87} \mathrm{Rb}$ condensate. We show that the classical seed is only relevant at low magnetic fields, where the amplified spin excitation mode has a large overlap with the original BEC. However, at larger magnetic fields the amplified spin excitations show pronounced spatial structures and lack a substantial overlap. As a result, our experiments show that the amplification is dominantly triggered by vacuum fluctuations. The amplification of vacuum fluctuations is a key requirement for the production of nonclassical matter waves with squeezed quadratures and strong quantum correlations. Our results hence open fascinating perspectives for spinor BECs, which may be employed in the near future as a source of two-mode squeezed matter waves and entangled Einstein-Podolski-Rosen pairs [13,14], and may possibly allow for Bell-type measurements [5,15] with neutral atoms. 
The onset of the spin dynamics is characterized by pair creation into $| \pm 1\rangle$. In order to analyze this initial regime, we consider the perturbed spinor $\hat{\mathbf{\Psi}}(\vec{r}, t)=\left[\boldsymbol{\Psi}_{0}(\vec{r})+\right.$ $\delta \hat{\boldsymbol{\Psi}}(\vec{r}, t)] e^{-i \mu t}$, where $\boldsymbol{\Psi}_{0}(\vec{r})=\left[0,0, n_{0}(\vec{r})^{1 / 2}, 0,0\right]^{T}$ represents the initial BEC in $|0\rangle, \mu$ is the chemical potential, and $\delta \hat{\boldsymbol{\Psi}}(\vec{r}, t)=\left(\delta \hat{\psi}_{-2}, \delta \hat{\psi}_{-1}, \delta \hat{\psi}_{0}, \delta \hat{\psi}_{1}, \delta \hat{\psi}_{2}\right)^{T} \quad$ describes fluctuations. Up to second order in $\delta \hat{\phi}_{ \pm}=\left(\delta \hat{\psi}_{+1} \pm\right.$ $\left.\delta \hat{\psi}_{-1}\right) / \sqrt{2}$, pair creation is described by the Hamiltonian $\hat{H}=\hat{H}_{+}+\hat{H}_{-}$, where

$$
\hat{H}_{ \pm}=\int d^{3} r\left[\delta \hat{\phi}_{ \pm}^{\dagger}\left(\hat{H}_{\text {eff }}+q\right) \delta \hat{\phi}_{ \pm} \pm \frac{\Omega}{2}\left(\delta \hat{\phi}_{ \pm}^{2}+\text { H.c. }\right)\right] .
$$

Here, $\quad \hat{H}_{\text {eff }}=-\hbar^{2} \nabla^{2} / 2 m+V_{\text {trap }}(\vec{r})+\left(U_{1}+U_{0}\right) n_{0}(\vec{r})-$ $\mu, V_{\text {trap }}(\vec{r})$ is the harmonic trap, $q \propto B^{2}$ characterizes the QZE, $\quad \Omega(\vec{r})=U_{1} n_{0}(\vec{r}), \quad$ and $\quad U_{0}=\left(7 g_{0}+10 g_{2}+\right.$ $\left.18 g_{4}\right) / 35$ and $U_{1}=\left(-7 g_{0}-5 g_{2}+12 g_{4}\right) / 35$ characterize the spin-preserving and spin-changing collisions. The coupling constant associated with the $s$-wave collisional channel with total spin $F$ is $g_{F}=4 \pi \hbar^{2} a_{F} / m$. Note that $\hat{H}_{ \pm}$is identical to the Hamiltonian describing an optical parametric amplifier [1].

The problem is analyzed best in the basis of eigenstates $\hat{H}_{\text {eff }} \varphi_{n}(\vec{r})=\epsilon_{n} \varphi_{n}(\vec{r})$. By introducing $\delta \hat{\phi}_{ \pm}=\sum_{n} \hat{b}_{n, \pm} \varphi_{n}$ and $\Omega_{n, n^{\prime}}=\int d^{3} r \Omega \varphi_{n} \varphi_{n^{\prime}}$ we may rewrite

$$
\hat{H}_{ \pm}=\sum_{n}\left(\epsilon_{n}+q\right) \hat{b}_{n, \pm}^{\dagger} \hat{b}_{n, \pm} \pm \sum_{n, n^{\prime}} \frac{\Omega_{n, n^{\prime}}}{2}\left(\hat{b}_{n, \pm} \hat{b}_{n^{\prime}, \pm}+\text { H.c. }\right) .
$$

The Heisenberg equation $i \hbar \frac{d}{d t} \hat{O}_{\nu}^{ \pm}=\left[H_{ \pm}, \hat{O}_{\nu}^{ \pm}\right]=\xi_{\nu}^{ \pm} \hat{O}_{\nu}^{ \pm}$ then yields the eigenvalues $\xi_{\nu}^{ \pm}$and the corresponding eigenoperators $\quad \hat{O}_{\nu}^{ \pm}=\sum_{n=1}^{M}\left(R_{\nu, n}^{ \pm} \hat{b}_{n, \pm}+R_{\nu, M+n}^{ \pm} \hat{b}_{n, \pm}^{\dagger}\right)$, where $M$ indicates the maximal $\varphi_{n}$ level considered.

Imaginary eigenvalues with $\operatorname{Im}\left(\xi_{\nu}\right)>0$ result in an exponential amplification of spin fluctuations, which indicates the onset of pair creation. In that case, the amplification dynamics is dominated by the most unstable mode $\nu_{0}$ with the largest imaginary part $\operatorname{Im}\left(\xi_{\nu_{0}}\right)=h \Lambda(q)$. The instability rate $\Lambda(q)$ generally shows a nonmonotonous multiresonant $q$ dependence due to the interplay between QZE, interactions, and external confinement [12].

Based on this diagonalization of the Hamiltonian, we can evaluate the experimentally relevant quantum evolution of the operators $\hat{a}_{n, m_{F}}$ and $\hat{a}_{n, m_{F}}^{\dagger}$, where $\hat{a}_{n, \pm 1}=$ $\left(\hat{b}_{n,+} \pm \hat{b}_{n,-}\right) / \sqrt{2}$. The total population in $| \pm 1\rangle$ is then $P_{m_{F}}(t)=\sum_{n}\left\langle\hat{a}_{n, m_{F}}^{\dagger}(t) \hat{a}_{n, m_{F}}(t)\right\rangle$, where the average is performed over the initial state $\left|\Psi_{m_{F}}(0)\right\rangle=|\Psi\rangle$.

In the presence of unstable spin excitation modes, the initial state $|\Psi\rangle$ triggers the subsequent amplification. To evaluate the triggering mechanism, the initial state $|\Psi\rangle$ including a classical seed can be represented by $|\Psi\rangle=$ $\left(N_{s} !\right)^{-1}\left(\hat{\Phi}_{+1}^{\dagger} \hat{\Phi}_{-1}^{\dagger}\right)^{N_{s}}|\mathrm{vac}\rangle$, where $\hat{\Phi}_{m_{F}}^{\dagger}\left(\hat{\Phi}_{m_{F}}\right)$ creates (annihilates) a $m_{F}$ particle in the mode of the initial BEC. In the basis of the effective Hamiltonian, they can be represented by a vector $\boldsymbol{\Phi}$ where $\hat{\Phi}_{m_{F}}=\sum_{n} \Phi_{n} \hat{a}_{n, m_{F}}$, with $\Phi_{n}=$ $\int d^{3} r \sqrt{n_{0}} \varphi_{n}$. Now, one obtains the total population as a sum of a classically and a quantum triggered contribution $P_{m_{F}}(t)=N_{S} P_{C}(t)+P_{Q}(t)$, where

$$
\begin{gathered}
P_{C}(t)=\boldsymbol{\Phi}^{*} \cdot\left[\hat{U}^{\dagger}(t) \cdot \hat{U}(t)+\hat{V}(t)^{\dagger} \cdot \hat{V}(t)\right] \cdot \boldsymbol{\Phi}, \\
P_{Q}(t)=\operatorname{Tr}\left\{\hat{V}^{\dagger}(t) \cdot \hat{V}(t)\right\},
\end{gathered}
$$

and the operators $\hat{U}(t)$ and $\hat{V}(t)$ are the time evolution operators according to $\hat{a}_{n, m_{F}}(t)=\sum_{n^{\prime}}\left[U_{n, n^{\prime}}(t) \hat{a}_{n^{\prime}, m_{F}}(0)+\right.$ $\left.V_{n, n^{\prime}}(t) \hat{a}_{n^{\prime},-m_{F}}^{\dagger}(0)\right]$.

We have employed Eqs. (1) and (2) to determine the population at any time $t$. Note, however, that $F=2{ }^{87} \mathrm{Rb}$ BECs present inherent hyperfine-changing losses [16]. Since these losses are small ( $<20 \%$ of the total number) during the typical investigation times of $20 \mathrm{~ms}$, they may be approximated by an experimentally determined exponential loss rate $\Gamma \simeq 10 \mathrm{~s}^{-1}$. The decreasing number of atoms results in a decreasing instability rate due to the reduced density as well as a slight change in the resonance condition as investigated in Ref. [12]. The loss has been incorporated in our analysis by a quasicontinuous reduction of the total atom number. Figure 1(b) shows the numerical result for our experimental parameters. Note that the multiresonant $q$ dependence of the instability rate $\Lambda(q)$ discussed in Ref. [12] directly maps into a multipeaked pair creation efficiency, which is in very good agreement with our previous experimental results [12] shown in Fig. 1(a). As we discuss in detail below, the unknown average classical seed $N_{s}$ only influences the spin dynamics significantly at the low-field resonance,

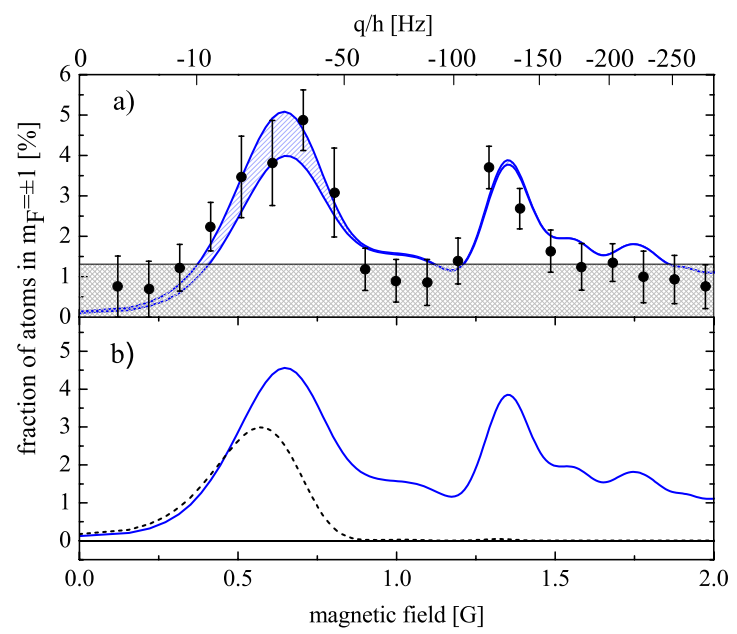

FIG. 1 (color online). (a) Fraction of atoms transferred into $\left|m_{F}= \pm 1\right\rangle$ within $21 \mathrm{~ms}$ as a function of the applied magnetic field [12]. The error bars indicate statistical uncertainties and the detection uncertainty. The shaded curve indicates the result of our theoretical prediction for a seed atom number of $N_{s}=2 \pm$ 0.4 , assuming an initial BEC in $|0\rangle$ with $N=50000$ (see text). The shaded box indicates the detection limit for a single atom number measurement. (b) Comparison of our calculation, including both the classical seed $N_{s}=2$ and vacuum spin fluctuations (solid) with the GP result (dashed). 
and hence it is obtained from a fit to the relative fraction of atoms on the two resonances. Our theoretical results for the amplification dynamics depend on the total number of atoms and especially on the precise values of the scattering lengths $a_{F}$ [17]. We varied these parameters within their rather strict uncertainties (1 standard deviation) obtaining a very good agreement with our experimental results (Fig. 1).

Figure 1(b) also shows that the spin dynamics differs significantly from the result of a simple mean-field GrossPitaevskii (GP) approach in which field operators $\hat{\psi}_{m_{F}}(\vec{r})$ are substituted by $c$-number fields $\psi_{m_{F}}(\vec{r})$. Quantum fluctuations are absent in this description. The striking difference between GP and exact results indicates that the two resonances display a very different sensitivity to a classical seed and vacuum fluctuations.

The relative importance of both triggering mechanisms is shown in Fig. 2 for an evolution time of $15 \mathrm{~ms}$ and for the limit of large evolution times. For large evolution times, only the most unstable mode contributes to the dynamics and we may approximate the evolution of $P_{C, Q}(t) \simeq$ $\bar{P}_{C, Q} \exp [2 \Lambda(q) t]$ and evaluate the time-independent ratio $\eta \stackrel{P}{=} \bar{P}_{C} / \bar{P}_{Q}$ between the classical and quantum-triggering mechanisms. For sufficiently small $|q|$, including the lowfield resonance shown in Fig. 1, any classical seed is highly relevant $(\eta \sim 1)$ due to the large overlap between the wave functions of the most unstable mode and the original BEC. However, for larger $|q|$, including the high-field resonance, the overlap is negligible and the population is dominantly triggered by vacuum fluctuations $(\eta \ll 1)$ [18]. Accordingly, thermal atoms are not amplified due to the negligible spatial overlap.

As detailed below, our experiments confirm this remarkable difference in sensitivity of the two resonances to any spurious classical seed. Note that this approach to determine the triggering mechanism avoids the difficulties arising in, e.g., the investigation of fluctuations of the amplifier output [19], which is largely impeded by the significant statistical uncertainty of the atom number measurement (detection noise) and small shot-to-shot variations of the

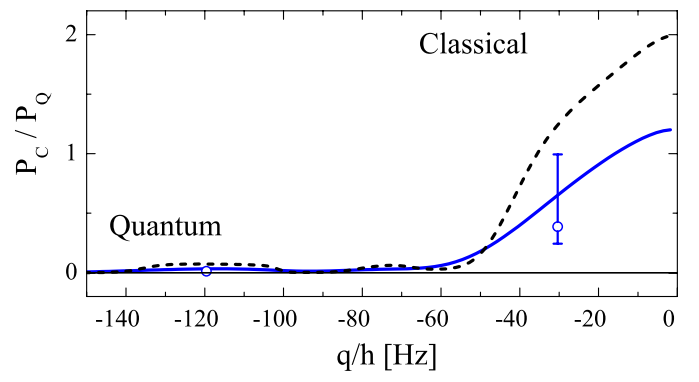

FIG. 2 (color online). Ratio $P_{C} / P_{Q}$ (see text) for an evolution time of $15 \mathrm{~ms}$ (solid) and for the limit of large evolution times (dashed). The quantum triggered dynamics is characterized by $P_{C} / P_{Q} \ll 1$. The hollow circles represent the experimental results. total atom number which lead to fluctuations in the pair creation efficiency (amplifier noise).

To initiate our experiments, we prepare BECs containing $7 \times 10^{4} F=2{ }^{87} \mathrm{Rb}$ atoms in the $|0\rangle$ state in an optical dipole trap with trapping frequencies of $(176,132,46) \mathrm{Hz}$. The effect of the thermal fraction (below 40\%) can be neglected due to its significantly smaller density. We carefully remove residual atoms in other spin components by briefly applying a strong magnetic field gradient of $\approx 50 \mathrm{G} / \mathrm{cm}$, which is ramped down within $10 \mathrm{~ms}$. To investigate the spin dynamics, the applied homogeneous magnetic field is subsequently lowered from $7.9 \mathrm{G}$ to a specific value between 0.12 and $2 \mathrm{G}$ within $3 \mathrm{~ms}$. The BEC is held at the chosen magnetic field for a variable time to allow for spin-changing collisions. Finally, the number of atoms in all $m_{F}$ components is measured independently by applying a strong magnetic field gradient during time-offlight expansion. To evaluate the onset of the amplification regime, we restrict our investigation to short spin evolution times and small populations in $| \pm 1\rangle$.

The sensitivity of the system to a classical seed is investigated by deliberately producing a very small symmetric seed population in the $| \pm 1\rangle$ states prior to the spin evolution. This is accomplished by using a rf pulse which transfers a variable number of atoms from the $|0\rangle$ to $| \pm 1\rangle$ states. This pulse is applied for $5 \mu \mathrm{s}$ at a magnetic field of 7.9 G. A frequency of $5.6 \mathrm{MHz}$ was identified, which couples the BEC in the $|0\rangle$ state symmetrically to the $| \pm 1\rangle$ states. The number of transferred atoms was calibrated at the smallest detectable numbers in the linear regime. Figure 3 shows a linear fit to the data which allows for an extrapolation to very small atom numbers. By reducing the rf power by a further 15 to $25 \mathrm{~dB}$, it is thus possible to reproducibly prepare a very small number of seed atoms. Note that both spuriously and deliberately produced seeds result from similar single-atom processes, and hence have exactly the same spatial dependence as the original BEC. The sensitivity to the deliberately produced seed is therefore representative of the sensitivity to any spurious seed in the experiment.

Figure 4(a) shows the fraction of atoms produced in the $| \pm 1\rangle$ states depending on the number of seed atoms for the

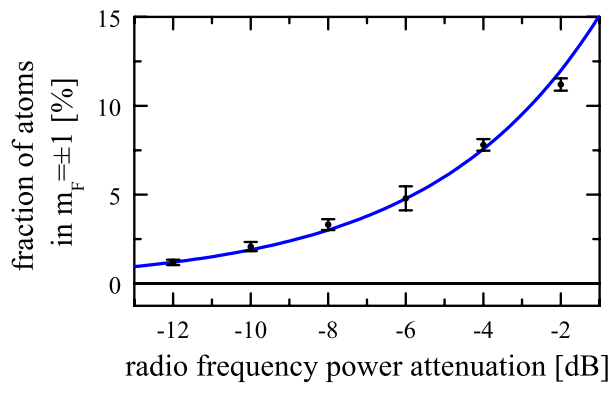

FIG. 3 (color online). Fraction of produced seed atoms in $\left|m_{F}= \pm 1\right\rangle$ as a function of the rf power on a logarithmic scale. The solid line indicates a linear fit to the data which allows for an extrapolation to very few seed atoms. 


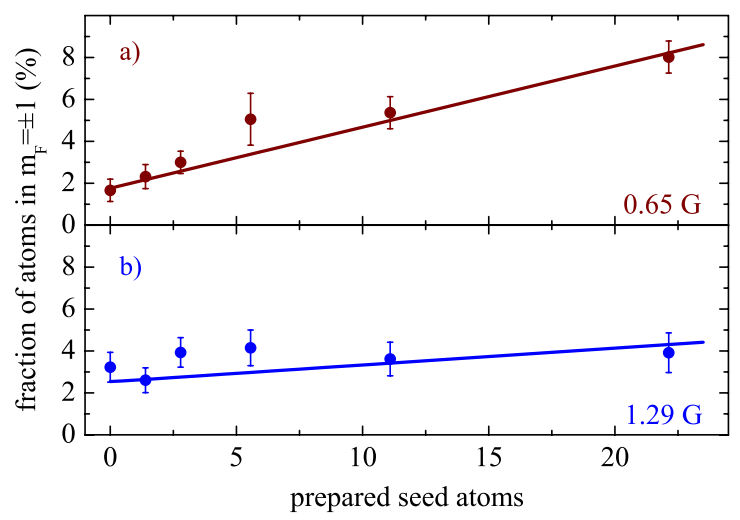

FIG. 4 (color online). Fraction of atoms transferred into $\left|m_{F}= \pm 1\right\rangle$ as a function of the number of deliberately prepared seed atoms. (a) The fraction on the low-field resonance at $0.65 \mathrm{G}$ shows a strong dependence on the classical seed after an evolution time of $15 \mathrm{~ms}$. (b) The fraction after $t=23 \mathrm{~ms}$ on the highfield resonance at $1.29 \mathrm{G}$ is basically independent of the number of seed atoms and we conclude that it is triggered by vacuum spin fluctuations. The error bars indicate statistical uncertainties. The solid lines represent the theory result with a seed atom number $N_{s}=4.5$.

low-field resonance (Fig. 1). Starting at a small offset value, this fraction grows linearly with an increasing number of seed atoms (an amplification of $23 \mathrm{~dB}$ ). Hence, the low-field resonance is strikingly sensitive to a classical seed, down to an extremely small number of seed atoms. The fact that the spin dynamics is sensitive to very few seed atoms also proves that the seed production works reliably and is not dominated by rf background noise. The offset is both due to the amplification of vacuum fluctuations and a small number of accidentally produced seed atoms. A comparison of our theoretical model yields a seed atom number of $N_{s}=4.5 \pm 0.3$, which is slightly higher than the value presented in Fig. 1 due to the additional preparation time after purification. An independent linear fit to the data yields the slope and the offset at zero seed atoms (at position $-N_{s}$ in the graph). The ratio of slope to offset yields the ratio of classical to quantum triggering. The resulting data points are shown in Fig. 2 and confirm our theoretical model.

However, this sensitivity to a classical seed is not general. Figure 4(b) shows the fraction of transferred atoms for the high-field resonance. For this resonance, the fraction of $| \pm 1\rangle$ atoms is largely independent of the number of deliberately produced seed atoms, which indicates that a spurious classical seed is irrelevant for sufficiently large $|q|$. Note, in particular, the remarkable difference between the results for $P_{C} / P_{Q}$ at both resonances in Fig. 2. This experimentally confirms that quantum triggering dominates the amplification dynamics at the high-field resonance and pair creation conclusively acts as a parametric amplification of vacuum fluctuations.

In conclusion, we have shown that a spinor $F=2$ BEC initially prepared in an unstable $|0\rangle$ state can provide a parametric amplifier for vacuum fluctuations, where the $|0\rangle$ BEC acts as an effective vacuum for atoms in $| \pm 1\rangle$. Similar to other parametric amplifiers, the system is exponentially sensitive to a spurious classical seed. We have therefore carefully analyzed the dependence of the pair creation efficiency on a classical seed in $| \pm 1\rangle$. For low magnetic fields, the amplification is extremely sensitive to spurious classical seed atoms, whereas for large enough fields the classical seed is irrelevant, and the observed pair creation is due to a parametric amplification of vacuum fluctuations. This mechanism is identical to spontaneous optical parametric down conversion and paves the way for the development of nonclassical atom optics on the basis of unstable spinor BECs. In particular, this system provides a direct path towards the observation of two-mode squeezing of matter waves, and a promising method for the creation of entangled atomic EPR pairs $[13,14]$. In addition, magnetic dipole-dipole interactions may play a significant role [20,21] for the case of $F=1$ BECs and will be the subject of future investigations.

We thank E. Tiemann and F. Deuretzbacher for fruitful discussions. We acknowledge support from the Centre for Quantum Engineering and Space-Time Research QUEST, and the European Science Foundation (EuroQUASAR).

[1] D. F. Walls and G. Milburn, Quantum Optics (Springer, Heidelberg, 1994).

[2] R. E. Slusher et al., Phys. Rev. Lett. 55, 2409 (1985).

[3] H. Vahlbruch et al., Phys. Rev. Lett. 95, 211102 (2005).

[4] Y.H. Shih and C. O. Alley, Phys. Rev. Lett. 61, 2921 (1988).

[5] A. Aspect, J. Dalibard, and G. Roger, Phys. Rev. Lett. 49, 1804 (1982).

[6] G. Weihs et al., Phys. Rev. Lett. 81, 5039 (1998).

[7] J.-W. Pan et al., Phys. Rev. Lett. 86, 4435 (2001).

[8] C. Orzel et al., Science 291, 2386 (2001).

[9] J. Esteve et al., Nature (London) 455, 1216 (2008).

[10] J. Hald, J. L. Sørensen, C. Schori, and E. S. Polzik, Phys. Rev. Lett. 83, 1319 (1999).

[11] A. Lamacraft, Phys. Rev. Lett. 98, 160404 (2007).

[12] C. Klempt et al., Phys. Rev. Lett. 103, 195302 (2009).

[13] L.-M. Duan, A. Sørensen, J. I. Cirac, and P. Zoller, Phys. Rev. Lett. 85, 3991 (2000).

[14] H. Pu and P. Meystre, Phys. Rev. Lett. 85, 3987 (2000).

[15] J. S. Bell, Physics 1, 195 (1964).

[16] H. Schmaljohann et al., Phys. Rev. Lett. 92, 040402 (2004).

[17] We use $a_{0}=87.9(2) a_{B}, a_{2}=91.2(2) a_{B}, a_{4}=99.0(2) a_{B}$ for the scattering lengths from a coupled channel analysis in the group of E. Tiemann.

[18] An equivalent analysis for Ref. [19] yields a quantumtriggering only for $q / h \leqslant 6 \mathrm{~Hz}$.

[19] S. R. Leslie et al., Phys. Rev. A 79, 043631 (2009).

[20] M. Vengalattore, S. R. Leslie, J. Guzman, and D. M. Stamper-Kurn, Phys. Rev. Lett. 100, 170403 (2008).

[21] T. Świsłocki, M. Brewczyk, M. Gajda, and K. Rzkażewski, Phys. Rev. A 81, 033604 (2010). 